\title{
Pycket: A Tracing JIT For a Functional Language
}

\author{
Spenser Bauman $^{a} \quad$ Carl Friedrich Bolz $^{b} \quad$ Robert Hirschfeld ${ }^{c}$ \\ Vasily Kirilichev ${ }^{c} \quad$ Tobias Pape $^{c} \quad$ Jeremy G. Siek $^{a} \quad$ Sam Tobin-Hochstadt $^{a}$ \\ ${ }^{a}$ Indiana University Bloomington, USA $\quad{ }^{b}$ King's College London, UK \\ ${ }^{c}$ Hasso Plattner Institute, University of Potsdam, Germany
}

\begin{abstract}
We present Pycket, a high-performance tracing JIT compiler for Racket. Pycket supports a wide variety of the sophisticated features in Racket such as contracts, continuations, classes, structures, dynamic binding, and more. On average, over a standard suite of benchmarks, Pycket outperforms existing compilers, both Racket's JIT and other highly-optimizing Scheme compilers. Further, Pycket provides much better performance for proxies than existing systems, dramatically reducing the overhead of contracts and gradual typing. We validate this claim with performance evaluation on multiple existing benchmark suites.

The Pycket implementation is of independent interest as an application of the RPython meta-tracing framework (originally created for PyPy), which automatically generates tracing JIT compilers from interpreters. Prior work on meta-tracing focuses on bytecode interpreters, whereas Pycket is a high-level interpreter based on the CEK abstract machine and operates directly on abstract syntax trees. Pycket supports proper tail calls and first-class continuations. In the setting of a functional language, where recursion and higher-order functions are more prevalent than explicit loops, the most significant performance challenge for a tracing JIT is identifying which control flows constitute a loop-we discuss two strategies for identifying loops and measure their impact.
\end{abstract}

\section{Introduction}

Contemporary high-level languages like Java, JavaScript, Haskell, ML, Lua, Python, and Scheme rely on sophisticated compilers to produce high-performance code. Two broad traditions have emerged in the implementation of such compilers. In functional languages, such as Haskell, Scheme, ML, Lisp, or Racket, ahead-of-time (AOT) compilers perform substantial static analysis, assisted by type information available either from the language's type system or programmer declarations. In contrast, dynamic, object-oriented languages, such as Lua, JavaScript, and Python, following the tradition of Self, are supported by just-in-time (JIT) compilers which analyze the execution of programs and dynamically compile them to machine code.

While both of these approaches have produced notable progress, the state of the art is not entirely satisfactory. In particular, for dynamically-typed functional languages, high performance traditionally requires the addition of type information, whether in the form of declarations (Common Lisp), type-specific operations (Racket), or an additional static type system (Typed Racket). Furthermore, AOT compilers have so far been unable to remove the overhead associated with highly-dynamic programming patterns, such as the dynamic checks used to implement contracts and gradual types. In these situations, programmers often make software engineering compromises to achieve better performance.
The importance of JavaScript in modern browsers has led to considerable research on JIT compilation, including both methodbased (Paleczny et al. 2001) and trace-based approaches (Gal et al. 2009). The current industry trend is toward method-based approaches, as the higher warm-up time for tracing JITs can have a greater impact on short-running JavaScript programs. However, for languages with different workloads, such as Python and Lua, tracing JITs are in widespread use (Bolz et al. 2009).

To address the drawbacks of AOT compilation for functional languages, and to explore a blank spot in the compiler design space, we present Pycket, a tracing JIT compiler for Racket, a dynamicallytyped, mostly-functional language descended from Scheme. Pycket is implemented using the RPython meta-tracing framework, which automatically generates a tracing JIT compiler from an interpreter written in RPython ("Restricted Python"), a subset of Python.

To demonstrate the effectiveness of Pycket, consider a function computing the dot product of two vectors in Racket:

$$
\text { (define (dot u v) (for/sum }([x u][y v])(* x y)) \text { ) }
$$

This implementation uses a Racket comprehension, which iterates in lockstep over $u$ and $v$, binding their elements to $x$ and $y$, respectively. The for/sum operator performs a summation over the values generated in each iteration, in this case the products of the vector elements. This dot function works over arbitrary sequences (lists, vectors, specialized vectors, etc) and uses generic arithmetic. Dot product is at the core of many numerical algorithms (Demmel 1997). We use this function as a running example throughout the paper.

In Racket, the generality of dot comes at a cost. If we switch from general to floating-point specific vectors and specialize the iteration and numeric operations, dot runs $6 \times$ faster. On the other hand, if we increase safety by adding contracts, checking that the inputs are vectors of floats, dot runs $2 \times$ slower.

In Pycket, the generic version runs at almost the same speed as the specialized version-the overhead of generic sequences, vectors, and arithmetic is eliminated. In fact, the code generated for the inner loop is identical-the performance differs only due to warm-up. Pycket executes the generic version of dot 5 times faster than the straightforwardly specialized version in Racket and 1.5 times faster than most manually optimized code we wrote for Racket. Furthermore, with the help of accurate loop-finding using dynamic construction of call graphs, Pycket eliminates the contract overhead in dot - the generated inner loop is identical to dot without contracts.

With Pycket, we depart from traditional Lisp and Scheme compilers in several of ways. First, we do no AOT optimization. The only transformations statically performed by Pycket are converting from core Racket syntax to A-normal form and converting assignments to mutable variables to heap mutations. We present an overview of Pycket's architecture and key design decisions in section 2. 
Second, Pycket performs aggressive run-time optimization by leveraging RPython's trace-based compilation facilities. With tracebased compilation, the runtime system starts by interpreting the program and watching for hot loops. Once a hot loop is detected, the system records the instructions executed in the loop and optimizes the resulting straight-line trace. Subsequent executions of the loop use the optimized trace instead of the interpreter. Thus, Pycket automatically sees through indirections due to pointers, objects, or higher-order functions. We present background on tracing in section 3 and describe optimizations that we applied to the interpreter that leverage tracing in section 5 .

Trace-based compilation poses a significant challenge for functional languages such as Racket because its only looping mechanism is the function call, but not all function calls create loops. Tracing JITs have used the following approaches to detect loops, but none of them is entirely adequate for functional languages.

- Backward Branches: Any jump to a smaller address counts as a loop (Bala et al. 2000). This approach is not suitable for an AST interpreter such as Pycket.

- Cyclic Paths: Returning to the same static program location counts as a loop (Hiniker et al. 2005). This approach is used in PyPy (Bolz et al. 2009), but in the context of Pycket, too many non-loop code paths are detected (Section 4).

- Static Detection: Identify loops statically, either from explicit loop constructs (Bebenita et al. 2010) or through static analysis of control flow (Gal and Franz 2006).

- Call Stack Comparison: To detect recursive functions, Hayashizaki et al. (2011) inspect the call stack to determine when the target of the current function call is already on the stack.

Section 4 describes our approach to loop detection that combines a simplified form of call-stack comparison with an analysis based on a dynamically constructed call graph.

Overall, we make the following contributions.

1. We describe the first high-performance JIT compiler for a dynamically typed functional language.

2. We show that tracing JIT compilation works well for eliminating the overhead of proxies and contracts.

3. We show that our combination of optimizations eliminates the need for manual specialization.

We validate these contributions with an empirical evaluation of each contribution in section 6. Our results show that Pycket is the fastest compiler among several mature, highly-optimizing Scheme systems such as Bigloo, Gambit, and Larceny on their own benchmark suite, and that its performance on contracts is substantially better than Racket, V8, and PyPy. Also, we show that manual specialization is not needed for good performance in Pycket. ${ }^{1}$

Pycket is available, together with links to our benchmarks, at

$$
\text { https://github.com/samth/pycket }
$$

\section{Pycket Primer}

This section presents the architecture of Pycket but defers the description of the JIT to section 3. Pycket is an implementation of Racket but is built in a way that generalizes to other dynamically typed, functional languages. Fundamentally, Pycket is an implementation of the CEK machine (Felleisen and Friedman 1987) but scaled up from the lambda calculus to most of Racket, including

\footnotetext{
1 This paper builds on unpublished, preliminary work presented by Bolz et al (2014). This paper reports major improvements with respect to performance, optimizations, loop-finding, coverage of the Racket language, and breadth of benchmarks, including programs with contracts.
}

$$
\begin{aligned}
& e::=x|\lambda x . e| e e \\
& \kappa::=[]|\arg (e, \rho):: \kappa| \operatorname{fun}(v, \rho):: \kappa \\
&\langle x, \rho, \kappa\rangle \longmapsto\langle\rho(x), \rho, \kappa\rangle \\
&\left\langle\left(e_{1} e_{2}\right), \rho, \kappa\right\rangle \longmapsto\left\langle e_{1}, \rho, \arg \left(e_{2}, \rho\right):: \kappa\right\rangle \\
&\left\langle v, \rho, \arg \left(e, \rho^{\prime}\right):: \kappa\right\rangle \longmapsto\left\langle e, \rho^{\prime}, \operatorname{fun}(v, \rho):: \kappa\right\rangle \\
&\left\langle v, \rho, \operatorname{fun}\left(\lambda x . e, \rho^{\prime}\right):: \kappa\right\rangle \longmapsto\left\langle e, \rho^{\prime}[x \mapsto v], \kappa\right\rangle
\end{aligned}
$$

Figure 1. The CEK Machine for the lambda calculus.

macros, assignment, multiple values, modules, structures, continuation marks and more. While this precise combination of features may not be present in many languages, the need to handle higherorder functions, dynamic data structures, control operators, and dynamic binding is common to languages ranging from OCaml to JavaScript.

Now to describe the implementation of Pycket, consider again the dot product function from the introduction:

(define (dot u v) (for/sum ( $\left.\left.\left[\begin{array}{cc}x & u\end{array}\right][y \quad v]\right)(* x y)\right)$ )

This example presents several challenges. First, for/sum and define are macros, which must be expanded to core syntax before interpretation. Second, these macros rely on runtime support functions from libraries, which must be loaded to run the function. Third, this loop is implemented with a tail-recursive function, which must avoid stack growth. We now describe our solutions to each of these challenges in turn.

Macros \& Modules Pycket uses Racket's macro expander (Flatt 2002) to evaluate macros, thereby reducing Racket programs to just a few core forms implemented by the runtime system (TobinHochstadt et al. 2011).

To run a Racket program, ${ }^{2}$ Pycket uses Racket to macro-expand all the modules used in a program and write the resulting forms and metadata JSON encoded files. Pycket then reads the serialized representation, parses it to an AST, and executes it. Adopting this technique enables Pycket to handle most of the Racket language while focusing on the key research contributions.

Assignment conversion and ANF Once a module is expanded to core Racket and parsed from the serialized representation, Pycket performs two transformations on the AST. First, the program is converted to A-normal form (ANF), ensuring that all non-trivial expressions are named (Danvy 1991; Flanagan et al. 1993). For example, converting the expression on the left to ANF yields the expression on the right.

$$
(*(+x 2)(+y 3))
$$

$$
\begin{gathered}
(\operatorname{let}((t 1 \quad(+x 2)) \\
(t 2(+y \quad 3))) \\
(* t 1 \quad t 2))
\end{gathered}
$$

Strictly speaking, converting to ANF might be characterized an AOT optimization in Pycket, however, the traditional use of ANF is not an optimization in itself, but as a simplified intermediate representation to enable further analysis and optimization, which Pycket does not do. We discuss below why ANF improves performance and the specific challenges in an interpreter only context.

Next, we convert all mutable variables (those that are the target of set!) into heap-allocated cells. This is a common technique in Lisp systems, and Racket performs it as well. This approach allows environments to be immutable mappings from variables to values, and localizes mutation in the heap. Additionally, each AST node stores its static environment; see section 5.1 for how this is used.

\footnotetext{
${ }^{2}$ Pycket supports programs written as modules but not an interactive REPL.
} 
CEK states and representation With our program in its final form, we now execute it using the CEK machine. To review, the CEK machine is described by the four transition rules in Figure 1. The CEK state has the form $\langle e, \rho, \kappa\rangle$ where $e$ is the AST for the program (the control), $\rho$ is the environment (a mapping of variables to values), and $\kappa$ is the continuation. A continuation is a sequence of frames and there are two kinds of frames: $\arg (e, \rho)$ represents the argument of a function application that is waiting to be evaluated and fun $(v, \rho)$ represents a function that is waiting for its argument. The first transition evaluates a variable by looking it up in the environment. The second and third transitions concern function applications; they reduce the function and argument expressions to values. The fourth transition performs the actual function call. Because no continuations are created when entering a function, tail calls are space efficient.

Initial execution of a program such as dot begins by injecting the expanded, assignment-converted and A-normalized program into a CEK machine triple with an empty continuation and environment The following is the (slightly simplified) main loop of the interpreter.

$$
\text { try: }
$$$$
\text { while True: }
$$$$
\text { ast, env, cont }=\text { ast. interpret (env, cont })
$$

except Done, e:

return e.values

This RPython code continuously transforms an (ast, env, cont) CEK triple into a new one, by calling the interpret method of the ast, with the current environment env and continuation cont as an argument. This process goes on until the continuation is the empty continuation, in which case a Done exception is raised, which stores the return value.

Environments and continuations are straightforward linked lists of frames, although environments store only values, not variable names, using the static environment information to aid lookup. Continuation frames are the frames from the CEK machine, extended to handle Racket's additional core forms such as begin and letrec.

Each continuation also contains information for storing continuation marks (Clements 2006), a Racket feature supporting stack inspection and dynamic binding. Because each continuation frame is represented explicitly and heap-allocated in the interpreter, rather than using the conventional procedure call stack, first-class continuations, as created by call/cc, are straightforward to implement, and carry very little run-time penalty, as the results of section 6 show. In this, our runtime representation resembles that of Standard ML of New Jersey (Appel and MacQueen 1991).

This approach also makes it straightforward to implement proper tail calls, as required by Racket and the Scheme standard (Sperber et al. 2010). On the other hand, one complication is that runtime primitives that call Racket procedures must be written in a variant of continuation-passing style, since each Racket-level function expects its continuation to be allocated explicitly and pushed on the continuation register. In contrast, typical interpreters written in RPython (and the Racket runtime system itself) expect user-level functions to return to their callers in the conventional way.

Contracts and Chaperones One distinctive feature of Racket is the extensive support for higher-order software contracts (Findler and Felleisen 2002). Software contracts allow programmers to specify preconditions, postconditions, and invariants using the programming language itself. This enables debugging, verification, program analysis, random testing, and gradual typing, among many other language features. Higher-order contracts allow greater expressiveness, scaling these features to modern linguistic constructs, but at the cost of wrappers and indirections, which often entail noticeable performance overhead. Contracts are also used in the implementation of gradual typing in Typed Racket (Tobin-Hochstadt and Felleisen
2008), where they protect the boundary between typed and untyped code. Here again, the cost of these wrappers has proved significant (St-Amour et al. 2012).

In Racket, contracts are implemented using the chaperone and impersonator proxying mechanism (Strickland et al. 2012), and make heavy use of Racket's structure system. These are the most complex parts of the Racket runtime system that Pycket supportsproviding comprehensive implementations of both. This support is necessary to run both the Racket standard library and most Racket programs. Our implementations of these features follow the highlevel specifications closely. In almost all cases, the tracing JIT compiler is nonetheless able to produce excellent results.

Primitives and values Racket comes with over 1,400 primitive functions and values; Pycket implements close to half of them. These range from numeric operations, where Pycket implements the full numeric tower including bignums, rational numbers, and complex numbers, to regular expression matching, to input/output including a port abstraction. As of this writing, more than half of the non-test lines of code in Pycket implement primitive functions.

One notable design decision in the implementation of primitive values is to abandon the Lisp tradition of pointer tagging. Racket and almost all Scheme systems, along with many other language runtimes, store small integers (in Racket, up to 63 bits on 64-bit architectures) as immediates, and only box large values, taking advantage of pointer-alignment restrictions to distinguish pointers from integers. Some systems even store other values as immediates, such as symbols, characters, or cons cells. Instead, all Pycket values are boxed, including small integers. This has the notable advantage that Pycket provides machine-integer-quality performance on the full range of machine integers, whereas systems that employ tagging will suffer performance costs for applications that require true 64-bit integers. However, abandoning tagging means relying even more heavily on JIT optimization-when the extra boxing cannot be optimized away, even simple programs perform poorly.

Limitations While Pycket is able to run a wide variety of existing Racket programs out of the box, it is not a complete implementation. The most notable absence is concurrency and parallelism: Racket provides threads, futures, places, channels, and events; Pycket implements none of these. Given the CEK architecture, the addition of threads (which do not use OS-level parallelism in Racket) should be straightforward. However, true parallelism in RPython-based systems remains a work in progress. Other notable absences include Racket's FFI, and large portions of the IO support, ranging from custom ports to network connectivity.

\section{Background on Tracing JITs and RPython}

Having described the basic architecture of Pycket, the next few sections explain how a high-level interpreter is turned into an optimizing JIT. We will again use the dot product from the introduction as an example.

A tracing JIT compiler optimizes a program by identifying and generating optimized machine code for the common execution paths. The unit of compilation for a tracing JIT is a loop, so a heuristic is needed to identify loops during interpretation. For the dot function, the identified loop is the tail-recursive function generated by the for/sum macro.

When a hot loop is identified, the JIT starts tracing the loop. The tracing process records the operations executed by the interpreter for one iteration of the loop. The JIT then optimizes the instruction sequence and generates machine code which will be used on subsequent iterations of the loop. During tracing, the JIT inserts guards into the trace to detect when execution diverges from the trace and needs to return control to the interpreter. Frequently taken fall back paths are also candidates for tracing and optimization. In 


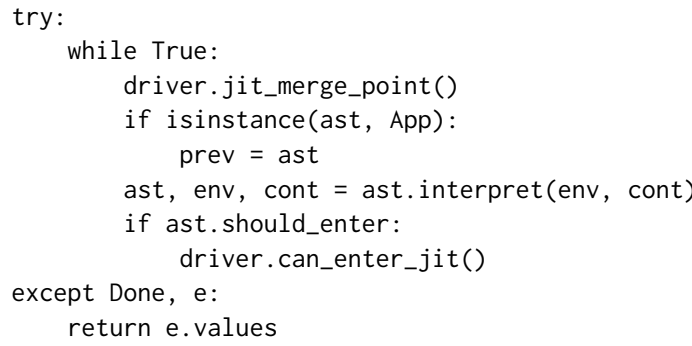

Figure 2. Interpreter main loop with hints

the dot function, guards are generated for the loop termination condition (one for each sequence). Additional tests, such as dynamic type tests, vector bounds checks, or integer overflow checks, are optimized away.

The RPython (Bolz et al. 2009; Bolz and Tratt 2013) project consists of a language and tool chain for implementing dynamic language interpreters. The RPython language is a subset of Python amenable to type inference and static optimization. The tool chain translates an interpreter, implemented in RPython, into an efficient virtual machine, automatically inserting the necessary runtime components, such as a garbage collector and JIT compiler. The translation process generates a control flow graph of the interpreter and performs type inference. This representation is then simplified to a low-level intermediate representation that is easily translated to machine code and is suitable for use in the tracing JIT.

Tracing JITs typically operate directly on a representation of the program; in contrast, the JIT generated by RPython operates on a representation of the interpreter; that is, RPython generates a metatracing JIT. To make effective use of the RPython JIT, the interpreter source must be annotated to help identify loops in the interpreted program, and to optimize away the overhead of the interpreter.

For Pycket, we annotate the main loop of the CEK interpreter as in figure 2. The annotations indicate that this is the main loop of the interpreter (jit_merge_point) and that AST nodes marked with should_enter are places where a loop in the interpreted program might start (can_enter_jit). At these places the JIT inspects the state of the interpreter by reading the local variables and then transfers control to the tracer.

In a conventional tracing JIT, loops can start at any target of a back-edge in the control-flow graph. In contrast, Pycket requires special care to determine where loops can start because the control flow of functional programs is particularly challenging to determine; see section 4 for the details.

\subsection{Generic RPython optimizations}

RPython backend performs a large number of trace optimizations. These optimizations are generic and not specialized to Pycket, but they are essential to understand the performance of Pycket.

Standard Optimizations RPython's trace optimizer includes a suite of standard compiler optimizations, such as commonsubexpression elimination, copy propagation, constant folding, and many others (Ardö et al. 2012). One advantage of trace compilation for optimization is that the linear control-flow graph of a trace is just a straight line. Therefore, the optimizations and their supporting analyses can be implemented in two passes over the trace, one forward pass and one backward pass.

Inlining Inlining is a vital compiler optimization for high-level languages, both functional and object-oriented. In a tracing JIT compiler such as RPython, inlining comes for free from tracing (Gal et al. 2006). A given trace will include the inlined code from any functions called during the trace. This includes Racket-level functions as well as runtime system functions (Bolz et al. 2009). The

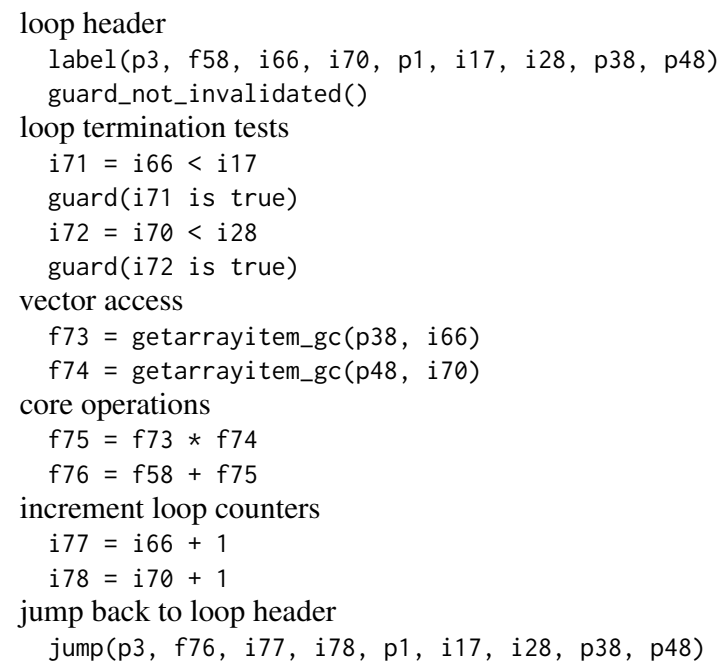

Figure 3. Optimized trace for dot inner loop

highly-aggressive inlining produced by tracing is one of the keys to its successful performance: it eliminates function call overhead and exposes opportunities for other optimizations.

Loop-invariant code motion Another common compiler optimization is loop-invariant code motion. RPython implements this in a particularly simple way, by peeling off a single iteration of the loop, and then performing its standard suite of forward analyses to optimize the loop further (Ardö et al. 2012). ${ }^{3}$ Because many loopinvariant computations are performed in the peeled iteration, they can then be omitted the second time, removing them from the actual loop body. In some cases, Pycket traces exposed weaknesses in the RPython JIT optimizer, requiring general-purpose improvements to the optimizer.

Allocation removal The CEK machine allocates a vast quantity of objects which would appear in the heap without optimization. This ranges from the tuple holding the three components of the machine state, to the environments holding each variable, to the continuations created for each operation. For example, a simple twoargument multiply operation, as found in dot, will create and use 3 continuation frames. Since both integers and floating-point numbers are boxed, unlike in typical Scheme implementations, many of these allocations must be eliminated to obtain high performance. Fortunately, RPython's optimizer is able to see and remove allocations that do not live beyond the scope of a trace (Bolz et al. 2011).

\subsection{An optimized trace}

The optimized trace for the inner loop of dot is shown in figure 3 Traces are represented in SSA form (Cytron et al. 1991). Variable names are prefixed according to type, with $p$ for pointers, $f$ for floats, and $i$ for integers. Several aspects of this trace deserve mention. First, the loop header and final jump appear to pass arguments, but this is merely a device for describing the content of memory - no function call is made here. Second, we see that there are two loop counters, as generated by the original Racket program. More sophisticated loop optimizations could perhaps remove one of them. Third, note that nothing is boxed-floating point values are stored directly in the array, the sum is stored in a register, as are the loop counters. This is the successful result of the allocation removal optimization. Third, note that no overflow, tag,

\footnotetext{
${ }^{3}$ Developed originally by Mike Pall in the context of LuaJIT.
} 
or bounds checks are generated. Some have been hoisted above the inner loop and others have been proved unnecessary. Finally, the guard_not_invalidated() call at the beginning of the trace does not actually become a check at run-time-instead, it indicates that some other operation might invalidate this code, forcing a recompile.

At points where control flow may diverge from that observed during tracing, guard operations are inserted. Guards take a conditional argument and behave as no-ops when that condition evaluates to true, while a false condition will abort the trace and hand control back to the interpreter. Many guards generated during tracing are elided by the trace optimizer; the only guards which remain in figure 3 encode the loop exit condition by first comparing the loop index (i66 and i70) to the length of each input array (i17 and i28). The guard operations then assert that each loop index is less than the array length.

Due to the design of Pycket as a CEK machine, Pycket relies on these optimizations much more heavily than PyPy. During tracing, many instructions which manage the CEK triple are recorded: allocating environments and continuation frames, building and destructing CEK triples, and traversing the environment to lookup variables. Allocation removal eliminates environments and continuations which do not escape a trace, constant folding and propagation eliminate management of the CEK triples, and the loop-invariant code motion pass eliminates environment lookups by hoisting them into a preamble trace. The result for the dot function is a tight inner loop without any of the original management infrastructure needed to manage the interpreter state.

\section{Finding Loops}

In Pycket, determining where loops start and stop is challenging. In a tracing JIT for a low-level language, the program counter is typically used to detect loops: a loop is a change of the program counter to a smaller value (Bala et al. 2000). Most RPython-based interpreters use a bytecode instruction set, where the same approach can still be used (Bolz et al. 2009).

However, Pycket, as a CEK machine, operates over the AST of the program, which is significantly more high-level than most bytecode instruction sets. The only AST construct which can lead to looping behavior is function application. Not every function application leads to a loop, so it is necessary to classify function applications into those than can close loops, and those that cannot.

One approach would be to perform a static analysis that looks at the program and tries to construct a call graph statically. This is, however, very difficult (i.e. imprecise) in the presence of higherorder functions and the possibility of storing functions as values in the heap. In this section, we describe two runtime approaches to detect appropriate loops, both of which are dynamic variants of the "false loop filtering" technique (Hayashizaki et al. 2011).

\subsection{Why Cyclic Paths are Not Enough}

In general, the body of every lambda may denote a trace header (i.e. may be the start of a loop). Though many functions encode loops, treating the body of every lambda as a trace header results in many traces that do not correspond to loops in the program text. For example, a non-recursive function called repeatedly in the body of a loop will initiate tracing at the top of the function and trace through its return into the body of the loop. Thus, identifying loops based on returning to the same static program location does not allow the JIT to distinguish between functions called consecutively ("false loops") and recursive invocations of a function. Consider the following example, which defines two functions $f$ and $g$, both of two arguments.

(define $(g a b)(+a b))$

(define ( $f a b)(g a b)(g a b)(f a b)$ )
The $g$ function computes the sum of its two arguments, while $f$ invokes $g$ on its arguments twice and then calls itself with the same arguments. Although it never terminates, $f$ provides a simple example of a false loop. $f$ forms a tail recursive loop, with two trace headers: at the beginning of the loops and at each invocation of $g$.

The function $g$ is invoked twice per iteration of $f$, so the JIT first sees $g$ as hot and begins tracing at one of the invocations of $g$. Tracing proceeds from the top of $g$ and continues until the interpreter next enters the body of $g$. This occurs by returning to the body of $f$ and tracing to the next invocation of g. As a result, only part of the loop body is traced. To cover the entire body of the loop, the guard generated to determine the return point of $\mathrm{g}$ must also be traced, encoding a single loop as multiple traces using guard operations to dispatch to the correct trace. This results in more time spent tracing and suboptimal code as the JIT cannot perform inter trace optimization.

\subsection{Two State Representation}

One method to "trick" the JIT into tracing a whole loop is to encode the location in the program as a pair of the current and previous AST node (with respect to execution). This distinction only matters at potential trace headers (the top of every lambda body). As such, each trace header is encoded as the body of the lambda along with its call site-only application nodes are tracked. The modified CEK loop (figure 2) stores this additional state in the prev variable, which is picked up by the JIT at the calls to the methods jit_merge_point and can_enter_jit.

In the example above, each invocation of $g$ would appear to the JIT as a separate location. Tracing will now begin at the first invocation of $g$, but will proceed through the second call and around the loop. Even though tracing did not begin at the "top" of the loop, the trace still covers a whole iteration. Such a "phase shift" of the loop has no impact on performance. This approach is a simplified version of the one proposed by Hayashizaki et al. (2011). Their solution looks at more levels of the stack to decide whether something is a false loop.

For recursive invocations of a function, this approach introduces little overhead as a function body will only have a few recursive call sites, though one trace will be generated for each recursive call site. Such functions may trace through several calls, as tracing will only terminate at a recursive call from the same call site which initiated the trace.

Detecting loops in this manner also has the potential to generate more traces for a given function: one per call site rather than just one for the function's body, as each trace is now identified by a call site in addition to the function which initiated the trace. Even in the presence of more precise loop detection heuristics, tracking the previous state is beneficial as the JIT will produce a larger number of more specialized traces.

\subsection{Call Graph}

When used in isolation, the approach described in the previous section does not produce satisfactory results for contracts. This is because the approach can be defeated by making calls go through a common call site, as in the following modified version of $g$ :

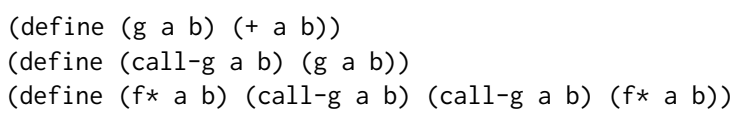

In such cases, the common call site hides the loop from the tracer, resulting in the same behavior as the original example. This behavior is a particular problem for contracts, leading to unsatisfactory results for code that uses them. Contracts use many extra levels of indirection and higher order functions in their implementation. 
Thus, the one extra level of context used by the two state approach is not consistently able to identify false loops.

To address this problem we developed a further technique. It uses a more systematic approach to loop detection that makes use of runtime information to construct a call graph of the program. A call graph is a directed graph with nodes corresponding to the source level functions in the program, and edges between nodes $A$ and $B$ if $A$ invokes $B$. We compute an under-approximation of the call graph at runtime. To achieve this, whenever the interpreter executes an application, the invoked function is inspected, and the lambda expression which created the function is associated with the lambda expression containing the current application. Thus portions of the full call graph are discovered incrementally.

Loop detection in the program then reduces to detecting cycles in the call graph. Cycle detection is also performed dynamically; when invoking a function, Pycket adds an edge to the call graph and checks for a cycle along the newly added edge. When a cycle is detected, a node in the cycle is annotated as a potential loop header, which the JIT will trace if it becomes hot. As opposed to the two state representation, the call graph is an opt-in strategy-initially, no AST nodes are potential trace headers. If the loop is generated by a tail-recursive loop, simple cycles are all the system needs to worry about. In the example above, $f *$ is the only cycle in the call graph and it is correctly marked as the only loop.

Non-tail-recursive loops are broken up by the CEK machine into multiple loops. Consider the following append function, which loops over its first argument, producing one continuation frame per cons cell.

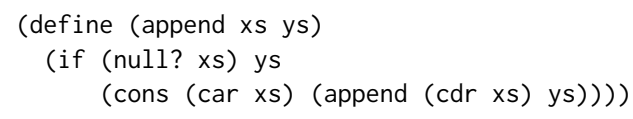

When append reaches the end of the first list, the accumulated continuation is applied to ys; the application of the continuation will continue, with each continuation cons-ing an element onto its argument. Thus, non-tail-recursive functions consist of a loop which builds up continuation frames (the "up" recursion) and a loop which applies continuation frames and performs outstanding actions (the "down" recursion).

To expose this fact to the JIT, the call graph also inspects the continuation after discovering a cycle. AST elements corresponding to continuations generated from the invoking function are marked in addition to the loop body. For the ANF version of the append example, the beginning of the function body would be marked as a loop for an "up" recursion, as would the body of the innermost let, containing the application of the cons function, which receives the result of the recursive call, performs the cons operation, and invokes the previous continuation. ${ }^{4}$

Though call graph recording and, in particular, cycle detection are not cheap operations, the performance gains from accurately identifying source level loops typically makes up for the runtime overhead. Due to the large amount of indirection, the call graph approach is necessary to obtain good performance with contracted code. Contracts can generate arbitrary numbers of proxy objects, so special care must be taken to avoid unrolling the traversal of deep proxies into the body of a trace. Such an unrolling generates sub optimal traces which are overspecialized on the depth of the proxied objects they operate on. Pycket uses a cutoff whereby operations on deep proxy stacks are marked as loops to be traced separately, rather than unrolled into the trace operating on the proxied object. This

\footnotetext{
4 A special case is primitive functions that are themselves loops, such as map. They must be marked in the interpreter source code so that the JIT generates a trace for them, even though there is no loop in the call graph.
}

produces a trace which loops over the proxy structure and dispatches any of the handler operations in the proxies.

Removal of the call graph leads to a slowdown of $28 \%$ across the full benchmark suite.

\section{Optimizing Interpreter Data Structures}

In this section, we describe a number of independent optimizations we applied to the data structures used by the Pycket interpreter, some novel (Section 5.1) and some taken from the literature (Section 5.2 and 5.3), which contribute significantly to overall performance. For each of these optimizations, we report how they impact performance by comparing Pycket in its standard mode (all optimizations on) to Pycket with the particular optimization off, across our full benchmark suite.

\subsection{Optimizing Environments in the presence of ANF}

As described in section 2 we translate all expressions to ANF prior to interpretation. This introduces additional let-bindings for all nontrivial subexpressions. Thus, function operands and the conditional expression of an if are always either constants, variables, or primitive operations that do not access the environment or the continuation, such as cons. The transformation to ANF is not required for our implementation, but significantly simplifies the continuations we generate, enabling the tracing JIT to produce better code.

Traditionally, ANF is used in the context of AOT compilers that perform liveness analysis to determine the lifetime of variables and make sure that they are not kept around longer than necessary. This is not the case in a naive interpreter such as ours. Therefore, ANF can lead to problems in the context of Pycket, since the inserted let-bindings can significantly extend the lifetime of an intermediate expression.

As an example of this problem, the following shows the result of transforming the append function to ANF:

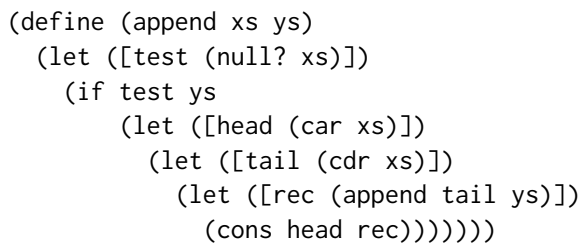

In the resulting code, (cdr xs) is live until the call to cons, whereas in the original code that value was only live until the recursive call to append. Even worse, the result of the test is live for the body of the append function. This problem is not unique to ANF-it can also affect code written by the programmer. However, ANF makes the problem more common, necessitating a solution in Pycket.

In Pycket, we counter the problem by attempting to drop environment frames early. Dropping an environment frame is possible when the code that runs in the frame does not access the variables of the frame at all, only those of previous frames. To this end, we do a local analysis when building a let AST. The analysis checks whether any of the outer environments of the let hold variables not read by the body of the let. If so, those environment frames are dropped when executing the let. In the example above, this is the case for the frame storing the tail variable, which can be dropped after the recursive call to append.

Additionally, if the parent of an environment frame is unreferenced, a new frame is created with just the child frame. This optimization produces an effect similar to closure conversion, ensuring that closures capture only used variables.

Disabling ANF entirely in the interpreter is not possible, but we can disable Pycket's environment optimizations. Across the full benchmark suite, Pycket is $3.5 \times$ faster when environment optimization is enabled. 


\subsection{Data Structure Specialization}

A number of interpreter-internal data structures store arrays of Racket values. Examples of these are environments and several kinds of continuations, such as those for let and function application. These data structures are immutable. ${ }^{5}$ Therefore, our interpreter chooses, at creation time, between specialized variants of these data structures optimized for the particular data it stores. Simple examination of the arguments to the constructor (all data structures are classes in RPython) suffices to choose the variant.

Pycket uses two kinds of variants. First, we specialize the data structures based on the number of elements, for all sizes between 0 and 10, inclusive. This avoids an indirection to a separate array to store the actual values. More importantly, there are several typespecialized variants. This helps to address the lack of immediate small integers (so-called fixnums), as mentioned in section 2. Boxed integers (and other boxed data) makes arithmetic slower, because the results of arithmetic operations must be re-boxed. This is mitigated by storing the fixnums' values directly in a specialized environment or continuation, without the need for an extra heap object.

All of these specializations come into play for the compilation of dot. The continuations and environments allocated all contain fewer than 10 values. Also, there are multiple environments that type-specialize based on their contents, such as one that holds the two integer loop counters, enabling further optimization by other parts of the trace optimizer.

In addition to the systematic specialization for continuations and environments, a few special cases of type specialization are directly coded in the representation of data structures. The most important example of these is cons cells that store fixnums in the car. This case again uses an unboxed representation to store the value. The optimization is made possible by the fact that Racket's cons cells are immutable. As an example, figure 4 shows the data layout of a type-specialized cons cell that is storing an unboxed fixnum.

These specializations combine for significant performance benefits. Across the full benchmark suite, Pycket with all optimizations produces a speedup of $17 \%$ over the version with type- and sizespecialization disabled.

\subsection{Strategies for Optimizing Mutable Objects}

Optimizing mutable objects by specialization is harder than optimizing immutable objects. When the content of the mutable object is changed, the specialized representation might not be applicable any more. Thus a different approach is needed to optimize mutable data structures such as Racket's vectors and hash tables.

For example, one would like to use a different representation for vectors that only store floating point numbers. In practice, many vectors are type-homogeneous in that way. Ideally the content of such a vector is stored in unboxed form, to save memory and make accessing the content quicker. However, because the vector is mutable, that representation needs to be changeable, for example if a value that is not a floating point number is inserted into the vector.

The RPython JIT specializes mutable collections using the storage strategies approach that was pioneered in the context of the PyPy project (Bolz et al. 2013). In that approach, the implementation of a collection object is split into two parts, its strategy and its storage. The strategy object describes how the contents of the collection are stored, and all operations on the collection are delegated to the strategy.

If a mutating operation is performed that needs to change the strategy, a new strategy is chosen and assigned to the collection. The storage is rewritten to fit what the new strategy expects. As an example, if a string is written to a vector using the unboxed

\footnotetext{
${ }^{5}$ Environments are immutable, despite the presence of set!, because of assignment conversion (section 2 ).
}

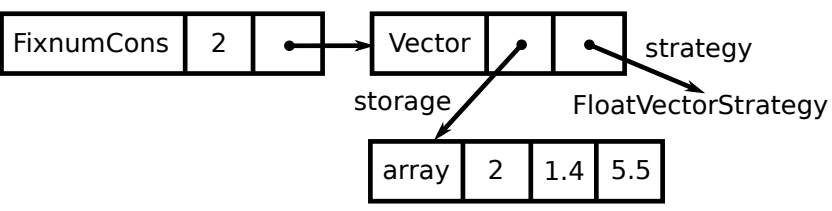

Figure 4. Optimized representation of (1 . \#(1.4 5.5)) using cons specialization and vector strategies

floating point strategy, a new strategy for generic Racket objects is chosen. New storage is allocated and the current vector's contents are boxed and moved into the new storage location. For a large vector, this is an expensive operation, and thus strategies depend for their performance on 1) the hypothesis that representation-changing mutations are rare on large data structures, and 2) the change to a more general strategy is a one-way street (Bolz et al. 2013).

Pycket uses strategies for the following kinds of objects: (a) vectors are type specialized if they contain all fixnums or all floatingpoint numbers (flonums); (b) hash tables are type specialized if their keys are all fixnums, bytes, symbols, or strings; (c) strings are specialized according to the kind of characters they store (Unicode or ASCII); (d) cells (used to store mutable variables) are type specialized for fixnums and flonums.

The use of strategies for vectors is a crucial optimization for dot. When the vectors are allocated with floating-point numbers, they use a strategy specialized to flonums, avoiding unboxing and tag checks for each vector reference in the inner loop. Racket programmers can manually do this type specialization by using flvectors, gaining back much of the lost performance. Pycket obviates the need for manual specialization by making generic vectors perform on par with specialized ones. As an example, figure 4 shows the data layout of a vector using a float strategy, with an array of unboxed floats as storage.

For hash maps the benefits are even larger than for vectors: if the key type is known, the underlying implementation uses a more efficient hashing and comparison function. In particular, because the comparison and hash function of these types is known and does not use arbitrary stack space or call/cc, the hash table implementation is simpler.

Strings are mutable in Racket, so they also use storage strategies. Since most strings are never mutated, a new string starts out with a strategy that the string is immutable. If later the string is mutated, it is switched to a mutable strategy. A further improvement of strings is the observation that almost all are actually ASCII strings, even though the datatype in Racket supports the full Unicode character range. Thus a more efficient ASCII strategy is used for strings that remain in the ASCII range. This makes them much smaller in memory (since every character needs only one byte, not four) and makes operations on them faster.

One special case of strategies is used for mutable heap cells which are used to implement mutable variables - those that are the target of set!. Quite often, the type of the value stored in the variable stays the same. Thus when writing a fixnum or a floating point number type into the cell, the cell switches to a special strategy that stores the values of these in unboxed form (bringing the usual advantages of unboxing).

Strategies are vital for high performance on benchmarks with mutable data structures. On dot, disabling strategies reduces performance by $75 \%$. For the benchmarks from section 6 that make extensive use of hash tables, disabling strategies makes some benchmarks 18 times slower. Over all benchmarks, the slowdown is $14 \%$. 


\section{Evaluation}

In this section, we evaluate Pycket's performance to test several hypotheses, as described in the introduction:

1. Meta-tracing JITs produce performance competitive with mature existing AOT compilers for functional languages.

2. Tracing JITs perform well for indirections produced by proxies and contracts.

3. Tracing JITs reduce the need for manual specialization.

To test the first hypothesis, we compare Pycket to Racket and 3 highly-optimizing Scheme compilers, across a range of Scheme benchmarks, and to Racket on benchmarks taken from the Racket repository. To test the second hypothesis, we measure Pycket and Racket's performance on both micro- and macro-benchmarks taken from the paper introducing Racket's chaperones and impersonators, the proxy mechanism underlying contracts, and also test V8 and PyPy on similar benchmarks. In particular, we show how the call graph based loop filtering of section 4.3 improves performance. To test the third hypothesis, we compare Pycket's performance on benchmarks with and without type specialization.

Our evaluation compares Pycket with multiple configurations and systems on a variety of programs. We present the most important results and include full results in supplemental material.

\subsection{Setup}

System We conducted the experiments on an Intel Xeon E5-2650 (Sandy Bridge) at $2.8 \mathrm{GHz}$ with $20 \mathrm{MB}$ cache and $16 \mathrm{~GB}$ of RAM. Although virtualized on Xen, the machine was idle. All benchmarks are single-threaded. The machine ran Ubuntu 14.04.1 LTS with a 64 bit Linux 3.2.0. We used the framework ReBench ${ }^{6}$ to carry out all measurements. RPython as of revision 954bf2fe475a was used for Pycket.

Implementations Racket v6.1.1 $\square$, Larceny v0.97 口, Gambit v4.7.2 , Bigloo v4.2a-alpha13Oct14 n, V8 v3.25.30 (and contracts.js ${ }^{7}$ version 0.2.0), PyPy v2.5.0, and Pycket $\square$ as of revision 535ee83 were used for benchmarking. Gambit programs were compiled with -D__SINGLE_HOST. Bigloo was compiled with -DLARGE_CONFIG to enable benchmarks to complete without running out of heap. In a few instances, Bigloo crashed, and in one case Gambit did not compile. These results were excluded from the average.

Methodology Every benchmark was run 10 times uninterrupted at highest priority in a new process. The execution time was measured in-system and, hence, does not include start-up; however, warm-up was not separated, so all times include JIT compilation. We show the arithmetic mean of all runs along with bootstrapped (Davison and Hinkley 1997) confidence intervals for a $95 \%$ confidence level.

Availability All of our benchmarks and infrastructure are available at http://gi thub.com/krono/pycket-bench.

\subsection{Benchmarks}

Larceny cross-platform benchmarks The benchmark suite consists of the "CrossPlatform" benchmark suite from Larceny, comprising well-known Scheme benchmarks originally collected for evaluating Gambit (about 27.7 KLOC in total). We increased iteration counts until Pycket took approximately 5 seconds, to lower jitter associated with fast-running benchmarks, and to ensure that we measure peak performance as well as JIT warmup (which is included in all measurements). Also, we moved all I/O out of the timed

\footnotetext{
${ }^{6}$ https://github.com/smarr/ReBench

${ }^{7}$ http://disnetdev. com/contracts. coffee/
}

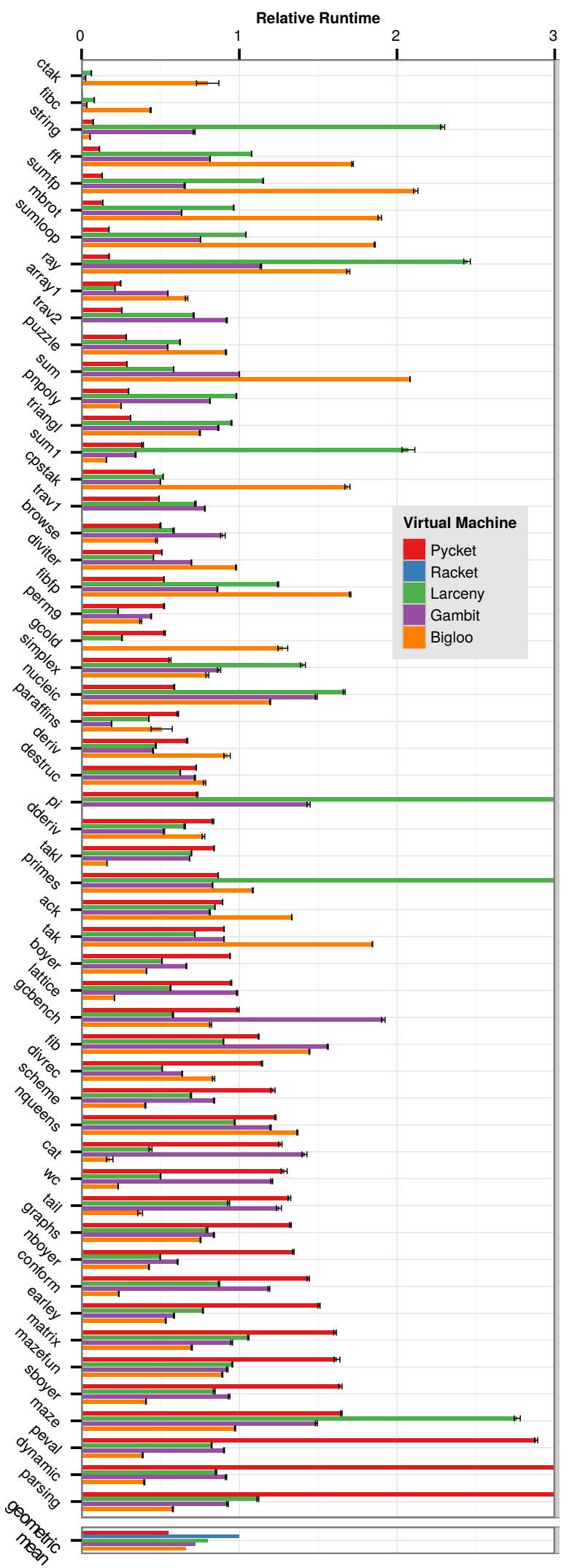

Figure 5. Scheme benchmarks, with runtimes normalized to Racket. Racket is omitted from the figure for clarity. Shorter is better. The geometric mean (including Racket) is shown at the bottom of the figure. 

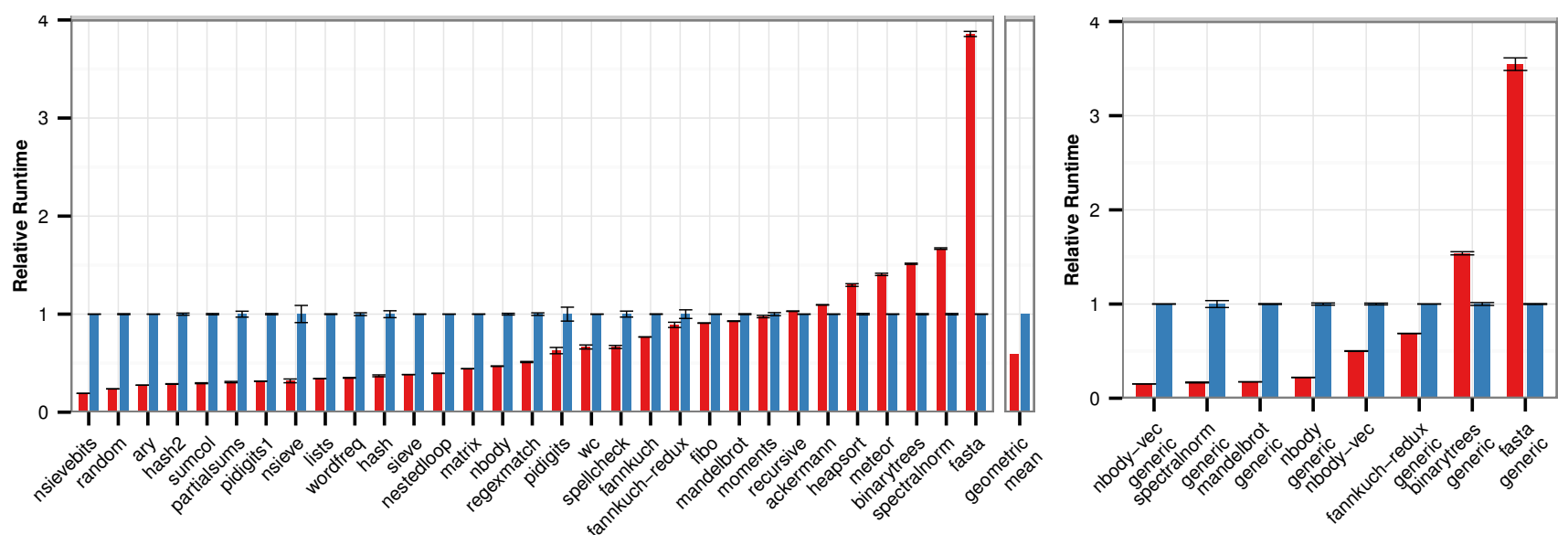

Figure 6. Racket benchmarks, runtimes normalized to Racket, consisting of those manually specialized for Racket (left) and generic versions with manual specialization removed (right). Lower is better.

loop, and omitted one benchmark (the slatex $\mathrm{LT}_{\mathrm{E}} \mathrm{X}$ preprocessor) where I/O was the primary feature measured.

The results are summarized in Figure 5. The runtime per benchmark of each system is normalized to Racket. The geometric mean of all measurements is given in bars at the right of the figure. The top of the chart cuts-off at 3 times the speed of Racket for space and readability, but some of the benchmarks on both Pycket and Larceny are between 3 and 4 times slower than Racket, and on two benchmarks ( $p i$ and primes, which stress bignum performance) Larceny is many times slower. Since first-class continuations are difficult to implement in a mostly-C environment, two benchmarks which focus on this feature ( $c t a k$ and $f i b c$ ) perform very poorly on Racket and Bigloo, both of which implement continuations by copying the C stack.

Pycket's performance on individual benchmarks ranges from approximately $4 \times$ slower to $470 \times$ faster than Racket; in 14 of 50 cases Pycket is the fastest implementation. On average, Pycket is the fastest system, and $45 \%$ faster than Racket.

Shootout benchmarks The traditional Scheme benchmark suite is useful for comparing across a variety of systems but often employ features and styles which are unidiomatic and less-optimized in Racket. Therefore, we also consider Racket-specific benchmarks written for the Computer Language Benchmarks Game (which are $1.9 \mathrm{KLOC}$ in total). ${ }^{8}$ We also include variants with manual specialization removed to demonstrate that Pycket can achieve good performance without manual specialization (600 LOC). The benchmarks are all taken from the Racket source repository, version 6.1.1. We omit two benchmarks, regexpdna and $k$-nucleotide, which require regular-expression features Pycket (and the underlying RPython library) does not support, and three, chameonos, thread-ring and echo, which require threading. We also modified one benchmark to move $\mathrm{I} / \mathrm{O}$ out of the main loop.

On average, Pycket is $44 \%$ faster than Racket on these benchmarks, is faster on 24 of the 31 , and is up to 5 times faster on numeric-intensive benchmarks such as random, nsievebits, or partialsums.

Chaperone benchmarks To measure the impact of our optimizations of contracts and proxies, we use the benchmarks created by Strickland et al. (2012). We run all of the micro-benchmarks (244 LOC) from that paper, and two of the macro-benchmarks $(1,438$

\footnotetext{
$\overline{{ }^{8} \text { http://shootout.alioth.debian.org/ }}$
}

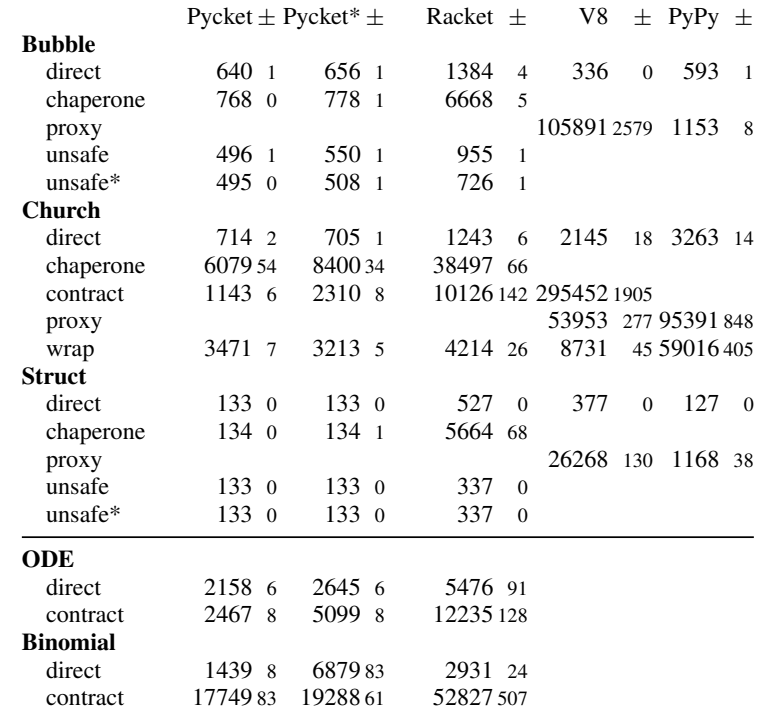

Table 1. Execution Times (in ms) for the Chaperone Benchmarks. Pycket* is Pycket without the call graph optimization

LOC). ${ }^{9}$ The results are presented in table 1 , with $95 \%$ confidence intervals. We show both Pycket's performance in the standard configuration (first column) as well as the performance without callgraphbased loop detection (second column).

The micro-benchmarks form the upper part of the table, with the macro-benchmarks below. For each micro-benchmark, we include representative implementations for V8 and PyPy. The Bubble benchmark is bubble-sort of a vector; Struct is structure access in a loop; and Church is calculating 9 factorial using Church numerals. The direct versions of all benchmarks omit proxying and contracts entirely. For Racket and Pycket, the chaperone versions use simple wrappers which are merely indirections, and the contract versions enforce invariants using Racket's contract library.

For V8, the proxy version uses JavaScript proxies (Van Cutsem and Miller 2010) and the contract version uses the contracts. coffee library (Disney et al. 2011), in both cases following the benchmarks

\footnotetext{
${ }^{9}$ All the other macro-benchmarks require either the FFI or the metaprogramming system at run-time, neither of which Pycket supports.
} 
conducted by Strickland et al. (2012). For PyPy, we implemented a simple proxy using Python's runtime metaprogramming facilities. The wrap version of the Church benchmark simply wraps each function in an additional function.

Two additional benchmark versions are also included for Racket and Pycket, labeled unsafe and unsafe*. The unsafe measurement indicates that specialized operations which skip safety checks were used in the program - in all cases, these can be justified by the other dynamic checks remaining in the program. The unsafe* measurement additionally assumes that operations are not passed instances of Racket's proxying mechanisms, an assumption that cannot usually be justified statically. The difference between these measurements is a cost paid by all optimized programs for the existence of proxies. In Pycket, this difference is much smaller than in Racket.

For the macro-benchmarks, we show results only for Pycket and Racket, with contracts on and off. The two macro-benchmarks are an ODE solver and a binomial heap, replaying a trace from a computer vision application, a benchmark originally developed for lazy contract checking (Findler et al. 2008). We show only the "opt chap" contract configuration of the latter, running on a trace from a picture of a koala, again following Strickland et al. (2012).

The results show that Pycket is competitive with other JIT compilers on micro-benchmarks, and that Pycket's performance on contracts and proxies is far superior both to Racket's and to other systems. In many cases, Pycket improves on other systems by factors of 2 to 100 , reducing contract overhead to almost 0 . Furthermore, the callgraph optimization is crucial to the performance of Pycket on contract-oriented benchmarks. Finally, the performance of PyPy on proxied arrays in the Bubble benchmark is noteworthy, suggesting both that tracing JIT compilers may be particularly effective at optimizing proxies and that Pycket's implementation combined with Racket's chaperone design provides additional benefits.

On the ODE benchmark, Pycket with the call graph provides a $6 \times$ speedup over Racket, resulting in less than $15 \%$ contract overhead. On the binomial heap benchmark, Pycket again outperforms Racket by a factor of 2, though substantial contract overhead remains, in all configurations. We conjecture the high contract overhead generally is due to the large numbers of proxy wrappers generated by this benchmark - as many as 2,800 on a single object. Racket's developers plan to address this by reducing the number of wrappers created in the contract system, ${ }^{10}$ which may allow Pycket to remove even more of the contract overhead.

Specialization benchmarks To measure Pycket's ability to eliminate the need for manual specialization, we constructed generic versions of several of the Racket benchmarks, and compared Racket and Pycket's performance. In all cases, Pycket's performance advantage over Racket improves. These results are presented in figure 5. Pycket loses only $6 \%$ of its performance when unspecialized, whereas Racket loses $30 \%$.

Warmup costs To understand the overhead that using a JIT instead of an AOT compiler adds to the execution of the benchmarks we measured the fraction of total benchmark execution time that is spent tracing, optimizing and producing machine code for each benchmark. Those fractions are shown in Figure 7. The results show that for almost all benchmarks the overhead of using a JIT is below $10 \%$. However, there are several outliers where code generation takes more than $50 \%$ of the runtime; we hope to address this in the future. Furthermore, this is not an issue for any program which runs longer than a few seconds. Figure 7 shows the time spent tracing and optimizing, but not the time spent interpreting code that is later JIT compiled or spent constructing the call graph, giving an underestimate of the total warmup time.

\footnotetext{
$\overline{{ }^{10} \text { Robby Findler, personal communication }}$
}

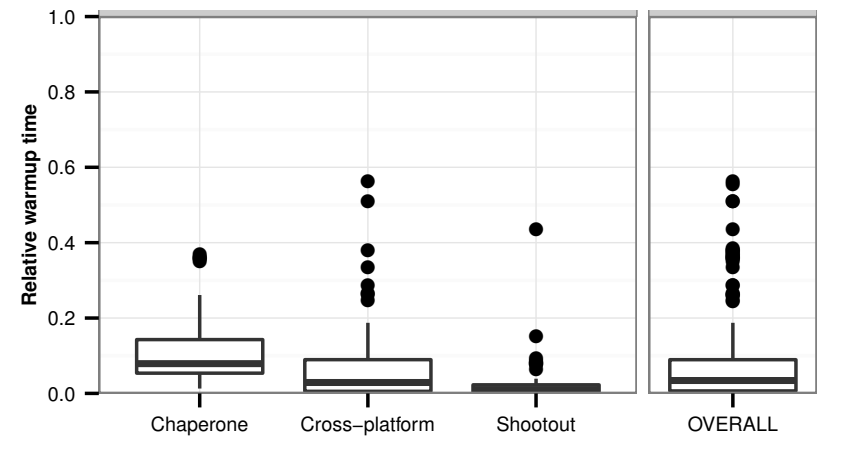

Figure 7. Boxplots of the runtime spent in the JIT as a fraction of the total runtime. The horizontal lines correspond to the 25th, 50th, and 75th percentile going from the bottom upward. The dots above each plot are outliers.

\subsection{Discussion}

Our evaluation results support all of our hypotheses. Pycket is faster than Racket across a broad range of benchmarks, and is competitive with highly-optimizing AOT compilers. Furthermore, Pycket can largely eliminate the need for manual specialization on types that is common in high-performance Racket and Scheme programs. Finally, call graph loop detection radically reduces the overhead of contracts, eliminating it entirely in some cases. In short, our tracing JIT is a success for Racket.

There are some cases where Pycket is substantially slower, specifically peval, dynamic, parsing and fasta. These cases are almost exclusively recursive programs with data-dependent control flow in the style of an interpreter over an AST. Such programs are a known weakness of tracing JIT compilers, although we hope to improve Pycket's performance in the future. Another important source of slowness is JIT warmup. Because Pycket is written in such a high-level style, the traces are much longer than for bytecode interpreters, which taxes the JIT optimizer. For a number of slow benchmarks, JIT optimizations and code generation take up a substantive portion of the benchmark runtime. We plan to address this issue both by modifying interpreter to be more low-level and by optimizing the RPython tracing infrastructure.

\section{Related Work}

As mentioned in the introduction, functional languages in general, and Scheme in particular, have a long tradition of optimizing AOT compilers. Rabbit, by Steele (1978), following on the initial design of the language, demonstrated the possibilities of continuationpassing style and of fast first-class functions. Subsequent systems such as Gambit (Feeley 2014), Bigloo (Serrano and Weis 1995), Larceny (Clinger and Hansen 1994), Stalin (Siskind 1999), and Chez (Dybvig 2011) have pioneered a variety of techniques for static analysis, optimization, and memory management, among others. Most other Scheme implementations are AST or bytecode interpreters. Racket is the only widely used system in the Scheme family with a JIT compiler, and even that is less dynamic than many modern JIT compilers and uses almost no runtime type feedback.

Many Lisp implementations, including Racket, provide means for programmers to manually optimize code with specialized operations or type declarations. We support Racket's type-specialized vectors and specialized arithmetic operations, as well as unsafe operations (e.g., eliding bounds checks). The results of our evaluation show that manual optimizations are less necessary in Pycket.

Type specialization is leveraged heavily in dynamic language interpreters to overcome the overhead of pointer tagging and boxing. 
There are two methods for generating type specialized code in the context of a JIT compiler: type inference and type feedback. Both methods have potential drawbacks; Kedlaya et al. (2013) explored the interaction between type feedback and type inference, using a fast, up front type inference pass to reduce the subsequent costs of type feedback. The performance impact of type information was studied in the context of ActionScript (Chang et al. 2011). Firefox's Spidermonkey compiler also uses a combination of type inference and type specialization (Hackett and Guo 2012).

In contrast, Pycket solely makes use of type specialization, which is a direct outcome of tracing. The RPython JIT has no access to type information of the Racket program aside from the operations recorded by the tracer during execution: a consequence of the JIT operating at the meta-level. In terms of type specialization, container strategies improve the effectiveness of type specialization by exposing type information about the contents of homogeneous containers to the tracer.

JIT compilation has been extensively studied in the context of object-oriented, dynamically typed languages (Aycock 2003). For Smalltalk-80, Deutsch and Schiffman (1984) developed a JIT compiler from bytecode to native code. Chambers et al. (1989) explored using type specialization and other optimizations in Self, a closely-related language. Further research on Self applied more aggressive type specialization (Chambers and Ungar 1991).

With the rise in popularity of Java, JIT compilation became a mainstream enterprise, with a significant increase in the volume of research. The Hotspot compiler (Paleczny et al. 2001) is representative of the Java JIT compilers. JIT compilation has also become an important topic in the implementation of JavaScript (see for example (Hölttä 2013)) and thus a core part of modern web browsers. For strict functional languages other than Scheme, such as OCaml, JIT compilers exist (Starynkevitch 2004; Meurer 2010), however, the AOT compilers for these languages are faster.

Mitchell (1970) introduced the notion of tracing JIT compilation, and Gal et al. (2006) used tracing in a Java JIT compiler. The core idea of meta-tracing, which is to trace an interpreter running a program rather than a program itself, was pioneered by Sullivan et al. (2003) in DynamoRIO. Since then, Gal et al. (2009) developed a tracing JIT compiler for JavaScript, TraceMonkey. LuaJIT ${ }^{11}$ is a very successful tracing JIT compiler for Lua. Further work was done by Bebenita et al. (2010) who created a tracing JIT compiler for Microsoft's CIL and applied it to a JavaScript implementation in C\#. These existing tracing systems, as well as PyPy and other RPython-based systems, differ from Pycket in several ways. First, they have not been applied to functional languages, which presents unique challenges such as first-class control, extensive use of closures, proper tail calls, and lack of explicit loops. Second, these systems all operate on a lower-level bytecode than Pycket's CEK machine, placing less burden on the optimizer. Third, few AOT compilers exist for these languages, making a head-to-head comparison difficult or impossible.

Schilling (2013, 2012) developed a tracing JIT compiler for Haskell based on LuaJIT called Lambdachine. Due to Haskell's lazy evaluation, the focus is quite different than ours. One goal of Lambdachine is to achieve deforestation (Wadler 1988; Gill et al. 1993) by applying allocation-removal techniques to traces.

There were experiments with applying meta-tracing to a Haskell interpreter written in RPython (Thomassen 2013). The interpreter also follows a variant of a high-level semantics (Launchbury 1993) of Core, the intermediate representation of the GHC compiler. While the first results were promising, it supports a very small subset of primitives leading to limited evaluation. It is unknown how well meta-tracing scales for a realistic Haskell implementation.

\footnotetext{
${ }^{11}$ http://luajit.org
}

\section{Conclusion}

Pycket is a young system-it has been under development for little more than a year, yet it is competitive with the best existing AOT Scheme compilers, particularly on safe, high-level, generic code, while still supporting complex features such as first-class continuations. Furthermore, Pycket is much faster than any other system on the indirections produced by contracts, addressing a widely noted performance problem, and making safe gradual typing a possibility in more systems.

The implementation of Pycket provides two lessons for JITs for functional languages. First, the issue of finding and exploiting loops requires careful consideration-explicit looping constructs in imperative languages make the tracer's life easier. Second, once this issue is addressed, conventional JIT optimizations such as strategies are highly effective in the functional context.

Our success in obtaining high performance from the CEK machine suggests that other high-level abstract machines may be candidates for a similar approach. Often language implementations sacrifice the clarity of simple abstract machines for lower-level runtime models-with a meta-tracing JIT such as RPython, the high-level approach can perform well. More generally, Pycket demonstrates the value of the RPython infrastructure (Marr et al. 2014): We have built in one year and 12,000 LOC a compiler competitive with existing mature systems. We encourage other implementors to consider if RPython can provide them with the same leverage.

Acknowledgements Bolz is supported by the EPSRC Cooler grant $\mathrm{EP} / \mathrm{K} 01790 \mathrm{X} / 1$. Siek is supported by NSF Grant 1360694 . We thank Anton Gulenko for implementing storage strategies.

\section{References}

A. Appel and D. MacQueen. Standard ML of New Jersey. In J. Maluszyński and M. Wirsing, editors, Programming Language Implementation and Logic Programming, volume 528 of Lecture Notes in Computer Science, pages 1-13. Springer Berlin Heidelberg, 1991.

H. Ardö, C. F. Bolz, and M. Fijakowski. Loop-aware optimizations in PyPy's tracing JIT. In Proceedings of the 8th Symposium on Dynamic Languages, DLS '12, pages 63-72, New York, NY, USA, 2012. ACM.

J. Aycock. A brief history of just-in-time. ACM Comput. Surv., 35(2):97-113, June 2003.

V. Bala, E. Duesterwald, and S. Banerjia. Dynamo: A transparent dynamic optimization system. In Proceedings of the ACM SIGPLAN 2000 Conference on Programming Language Design and Implementation, PLDI '00, pages 1-12, New York, NY, USA, 2000. ACM.

M. Bebenita, F. Brandner, M. Fahndrich, F. Logozzo, W. Schulte, N. Tillmann, and H. Venter. SPUR: A trace-based JIT compiler for CIL. In Proc. OOPSLA, pages 708-725, 2010.

C. F. Bolz and L. Tratt. The impact of meta-tracing on VM design and implementation. Science of Computer Programming, 2013.

C. F. Bolz, A. Cuni, M. Fijakowski, and A. Rigo. Tracing the meta-level: PyPy's tracing JIT compiler. In Proc. ICOOOLPS, pages 18-25, 2009.

C. F. Bolz, A. Cuni, M. Fijakowski, M. Leuschel, S. Pedroni, and A. Rigo. Allocation removal by partial evaluation in a tracing JIT. Proc. PEPM, pages 43-52, 2011.

C. F. Bolz, L. Diekmann, and L. Tratt. Storage strategies for collections in dynamically typed languages. In Proc. OOPSLA, pages 167-182, 2013.

C. F. Bolz, T. Pape, J. G. Siek, and S. Tobin-Hochstadt. Meta-tracing makes a fast Racket. In Workshop on Dynamic Languages and Applications, 2014.

C. Chambers and D. Ungar. Iterative type analysis and extended message splitting: optimizing dynamically-typed object-oriented programs. Lisp Symb. Comput., 4(3):283-310, 1991.

C. Chambers, D. Ungar, and E. Lee. An efficient implementation of SELF a dynamically-typed object-oriented language based on prototypes. In Proc. OOPSLA, pages 49-70, 1989. 
M. Chang, B. Mathiske, E. Smith, A. Chaudhuri, A. Gal, M. Bebenita, C. Wimmer, and M. Franz. The impact of optional type information on JIT compilation of dynamically typed languages. In Symposium on Dynamic Languages, DLS '11, pages 13-24, 2011.

J. Clements. Portable and high-level access to the stack with Continuation Marks. PhD thesis, Citeseer, 2006.

W. D. Clinger and L. T. Hansen. Lambda, the ultimate label or a simple optimizing compiler for Scheme. In Proc. LFP, pages 128-139, 1994.

R. Cytron, J. Ferrante, B. K. Rosen, M. N. Wegman, and F. K. Zadeck. Efficiently computing static single assignment form and the control dependence graph. ACM Transactions on Programming Languages and Systems, 13(4):451490, Oct. 1991.

O. Danvy. Three steps for the CPS transformation. Technical Report CIS 92-02, Kansas State University, 1991.

A. C. Davison and D. V. Hinkley. Bootstrap Methods and Their Application, chapter 5. Cambridge, 1997.

J. W. Demmel. Applied Numerical Linear Algebra. SIAM, 1997.

L. P. Deutsch and A. M. Schiffman. Efficient implementation of the Smalltalk-80 system. In Proc. POPL, pages 297-302, 1984.

T. Disney, C. Flanagan, and J. McCarthy. Temporal higher-order contracts. In Proceedings of the 16th ACM SIGPLAN International Conference on Functional Programming, ICFP'11, pages 176-188, New York, NY, USA, 2011. ACM.

R. K. Dybvig. Chez Scheme version 8 user's guide. Technical report, Cadence Research Systems, 2011.

M. Feeley. Gambit-C: A portable implementation of Scheme. Technical Report v4.7.2, Universite de Montreal, February 2014.

M. Felleisen and D. P. Friedman. Control operators, the SECD-machine and the lambda-calculus. In Working Conf. on Formal Description of Programming Concepts - III, pages 193-217. Elsevier, 1987.

R. B. Findler and M. Felleisen. Contracts for higher-order functions. In International Conference on Functional Programming, ICFP, pages 4859, October 2002.

R. B. Findler, S.-y. Guo, and A. Rogers. Lazy contract checking for immutable data structures. In Implementation and Application of Functional Languages, pages 111-128. Springer, 2008.

C. Flanagan, A. Sabry, B. F. Duba, and M. Felleisen. The essence of compiling with continuations. In Proc. PLDI, pages 502-514, 1993.

M. Flatt. Composable and compilable macros:: you want it when? In ICFP '02: Proceedings of the seventh ACM SIGPLAN international conference on Functional programming, pages 72-83. ACM Press, 2002.

A. Gal and M. Franz. Incremental dynamic code generation with trace trees. Technical Report ICS-TR-06-16, University of California, Irvine, 2006.

A. Gal, C. W. Probst, and M. Franz. HotpathVM: an effective JIT compiler for resource-constrained devices. In Proc. VEE, pages 144-153, 2006.

A. Gal, B. Eich, M. Shaver, D. Anderson, D. Mandelin, M. R. Haghighat, B. Kaplan, G. Hoare, B. Zbarsky, J. Orendorff, J. Ruderman, E. W. Smith, R. Reitmaier, M. Bebenita, M. Chang, and M. Franz. Trace-based justin-time type specialization for dynamic languages. In Proc. PLDI, pages 465-478. ACM, 2009.

A. Gill, J. Launchbury, and S. L. Peyton Jones. A short cut to deforestation. In Proc. FPCA, pages 223-232, 1993.

B. Hackett and S.-y. Guo. Fast and precise hybrid type inference for javascript. In Proceedings of the 33rd ACM SIGPLAN Conference on Programming Language Design and Implementation, PLDI'12, pages 239-250, New York, NY, USA, 2012. ACM.

H. Hayashizaki, P. Wu, H. Inoue, M. J. Serrano, and T. Nakatani. Improving the performance of trace-based systems by false loop filtering. In Proceedings of the Sixteenth International Conference on Architectural Support for Programming Languages and Operating Systems, ASPLOS XVI, pages 405-418, New York, NY, USA, 2011. ACM.

D. Hiniker, K. Hazelwood, and M. D. Smith. Improving region selection in dynamic optimization systems. In Proceedings of the 38th Annual IEEE/ACM International Symposium on Microarchitecture, MICRO 38, pages 141-154. IEEE Computer Society, 2005.
M. Hölttä. Crankshafting from the ground up. Technical report, Google, August 2013.

M. N. Kedlaya, J. Roesch, B. Robatmili, M. Reshadi, and B. Hardekopf. Improved type specialization for dynamic scripting languages. In Proceedings of the 9th Symposium on Dynamic Languages, DLS '13, pages 37-48. ACM, 2013.

J. Launchbury. A natural semantics for lazy evaluation. In Proc. POPL, pages 144-154, 1993.

S. Marr, T. Pape, and W. De Meuter. Are we there yet? Simple language implementation techniques for the 21st century. IEEE Software, 31(5): 6067, Sept. 2014.

B. Meurer. OCamlJIT 2.0 - Faster Objective Caml. CoRR, abs/1011.1783, 2010.

J. G. Mitchell. The Design and Construction of Flexible and Efficient Interactive Programming Systems. PhD thesis, Carnegie Mellon University, 1970.

M. Paleczny, C. Vick, and C. Click. The Java Hotspot server compiler. In Proc. JVM, pages 1-1. USENIX Association, 2001.

T. Schilling. Challenges for a Trace-Based Just-In-Time Compiler for Haskell. In Implementation and Application of Functional Languages, volume 7257 of $L N C S$, pages 51-68. 2012.

T. Schilling. Trace-based Just-in-time Compilation for Lazy Functional Programming Languages. PhD thesis, University of Kent, 2013.

M. Serrano and P. Weis. Bigloo: a portable and optimizing compiler for strict functional languages. In Static Analysis, volume 983 of LNCS, pages 366-381. 1995.

J. M. Siskind. Flow-directed lightweight closure conversion. Technical Report 99-190R, NEC Research Institute, Inc., 1999.

M. Sperber, R. K. Dybvig, M. Flatt, A. van Straaten, R. Findler, and J. Matthews. Revised ${ }^{6}$ Report on the Algorithmic Language Scheme. Cambridge, 2010.

V. St-Amour, S. Tobin-Hochstadt, and M. Felleisen. Optimization coaching Optimizers learn to communicate with programmers. In Proceedings of the ACM International Conference on Object Oriented Programming Systems Languages and Applications, OOPSLA '12, pages 163-178, New York, NY, USA, 2012. ACM.

B. Starynkevitch. OCAMLJIT - A faster just-in-time OCaml implementation. In First MetaOCaml Workshop, Oct. 202004.

G. L. Steele. Rabbit: A compiler for Scheme. Technical Report AI-474, MIT, 1978.

T. S. Strickland, S. Tobin-Hochstadt, R. B. Findler, and M. Flatt. Chaperones and impersonators: run-time support for reasonable interposition. In Object Oriented Programming Systems Languages and Applications, OOPSLA'12, 2012.

G. T. Sullivan, D. L. Bruening, I. Baron, T. Garnett, and S. Amarasinghe. Dynamic native optimization of interpreters. In Proc. IVME, pages 50-57, 2003.

E. W. Thomassen. Trace-based just-in-time compiler for Haskell with RPython. Master's thesis, Norwegian University of Science and Technology Trondheim, 2013.

S. Tobin-Hochstadt and M. Felleisen. The design and implementation of typed scheme. In Proceedings of the 35th Annual ACM SIGPLANSIGACT Symposium on Principles of Programming Languages, POPL '08, pages 395-406, New York, NY, USA, 2008. ACM.

S. Tobin-Hochstadt, V. St-Amour, R. Culpepper, M. Flatt, and M. Felleisen Languages as libraries. In Proceedings of the 32nd ACM SIGPLAN conference on Programming language design and implementation, PLDI '11, pages 132-141. ACM, 2011.

T. Van Cutsem and M. S. Miller. Proxies: Design principles for robust object-oriented intercession apis. In Proceedings of the 6th Symposium on Dynamic Languages, DLS '10, pages 59-72, New York, NY, USA, 2010. ACM.

P. Wadler. Deforestation: transforming programs to eliminate trees. In Proc. ESOP, pages 231-248, 1988. 